\title{
Biliary Tract Infection, CTCAE
}

National Cancer Institute

\section{Source}

National Cancer Institute. Biliary Tract Infection, CT CAE. NCI Thesaurus. Code C143317.

A disorder characterized by an infectious process involving the biliary tract. 